\title{
Pericardial mesothelioma
}

A 68-year-old man presented with symptoms of dyspnoea, productive cough, profound lethargy, weight loss and hematemesis. He was a retired heating engineer with a history of asbestos exposure and 2 years previously underwent pleural biopsy that confirmed epitheloid mesothelioma. His chest radiograph showed left lower lobe consolidation, a pleural effusion and increased cardiothoracic ratio. He underwent echocardiography that showed a possible thrombus and he was referred for a cardiac magnetic resonance.

Cardiac magnetic resonance imaging revealed meditational shift with a large left-sided mass infiltrating the pericardium, measuring $4.7 \times 3 \mathrm{~cm}$ over the apical lateral left ventricle and right ventricle free wall. T2-weighted images denoted pericardial infiltration with high signal intensity relative to the myocardium (Figure 1). Contrast-enhanced images indicated a laminar thrombus in left ventricular apex, as well as signs of necrosis and haemorrhage in the pericardial infiltrate. An ejection fraction of $63 \%$ was recorded with no suggestion of pericardial constriction. This patient deteriorated with increasing fatigue and breathlessness and died 3 weeks later.

Malignant mesothelioma is a rapidly fatal epithelial neoplasm that arises from the pleura, peritoneum and pericardium.
Clinicians need to be aware that $80 \%$ of patients have a history of asbestos exposure, and prolonged exposure is associated with a shorter latency period to disease development. Following prediction from levels of asbestos exposure in the 20th century, peak of mortality from malignant mesothelioma should span from 2011 to 2015, and a decline in death rate should now be observed. Despite this, cases are still seen and early diagnosis results in improved outcome.

\section{Funding}

The authors acknowledge financial support from the Department of Health, via the National Institute for Health Research (NIHR) comprehensive Biomedical Research Centre award to Guy's \& St Thomas' NHS Foundation Trust in partnership with King's College London and King's College Hospital NHS Foundation Trust. J Webb has received a research grant from EU FP7 (number 611823).

Photographs and text from: Jessica Webb, Division of Imaging Sciences and Biomedical Engineering, King's College
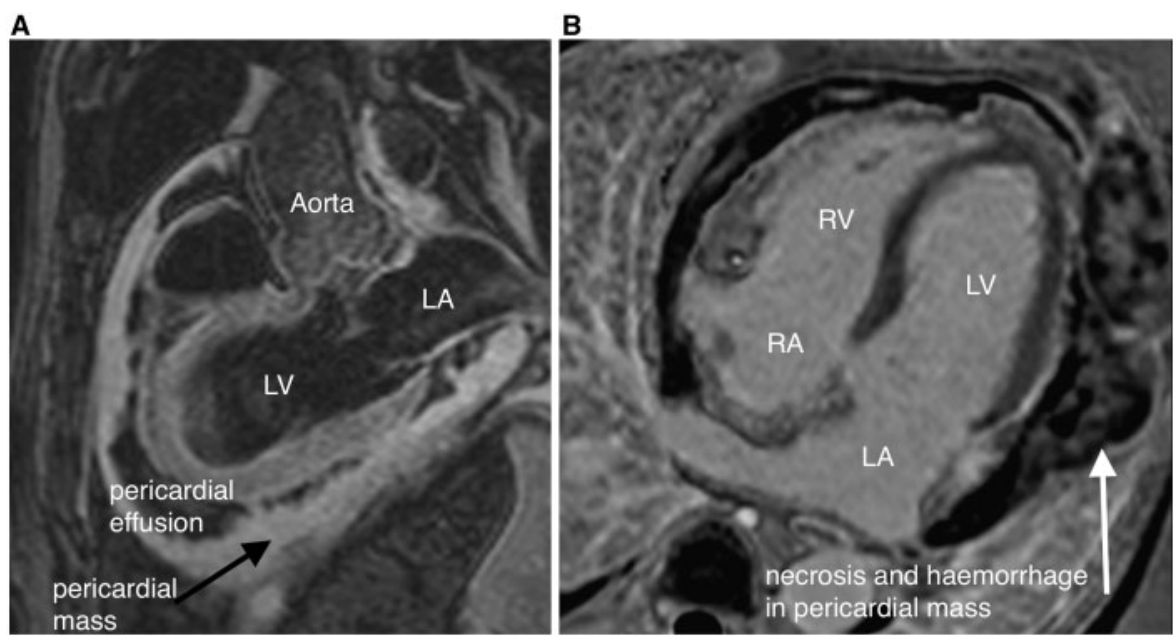

Figure 1. (A) Three-chamber, T2-weighted image showing large circumferential pericardial mass (black arrow) with pericardial effusion (PE). Left atrium (LA), left ventricle (LV) and aorta are marked. (B) Four-chamber image following late gadolinium enhancement, showing areas of necrosis and haemorrhage within the pericardial mass (marked with white arrow). LV, LA, right ventricle (RV) and right atrium (RA) are marked. 
632 | QJM: An International Journal of Medicine, 2016, Vol. 109, No. 9

London, London SE1 7EH, UK and Department of Cardiology, St Thomas' Hospital, London SE1 7EH, UK; Y.W. Yiu, Division of Imaging Sciences and Biomedical Engineering, King's College London, London SE1 7EH, UK; S. Giastefani, Department of Cardiology, Croydon University Hospital, Croydon, Surrey CR7
7YE, UK; G. Carr-White, Department of Cardiology, St Thomas' Hospital, London SE1 7EH, UK. email: Jessica.webb@kcl.ac.uk

Conflict of interest: None declared. 